\title{
El fútbol callejero como herramienta educativa de transformación social en la comuna de Pedro Aguirre Cerda: organización Pac Gol
}

\author{
Juan Ignacio Gutiérrez Martinoli ${ }^{1}$
}

\begin{abstract}
RESUMEN
El artículo analiza cómo la metodología del fútbol callejero (MFC) fortalece la construcción identitaria de los jóvenes participantes de la organización comunitaria Pac Gol, ubicada en la comuna de Pedro Aguirre Cerda, desde un modelo de educación popular que confronta la reproducción de estereotipos sexuales, raciales y de clase presente en el modelo de educación formal y en dimensiones del fútbol tradicional. Se emplea una metodología cualitativa y de corte trasversal, que combina la realización de entrevistas semiestructuradas con observación participante. Se logró, por un lado, identificar el arraigo de sus miembros respecto de la organización - expresado tanto en lazos afectivos de confianza recíproca entre sus actores como en la apropiación territorial y el bienestar subjetivo de los sujetos- $y$, por otro, la evidencia sobre cambios de pautas, normas y actitudes en los jugadores participantes, en torno a valores relativos a la inclusión social y la no discriminación.
\end{abstract}

Palabras clave: identidad, educación popular, fútbol callejero, capital social

\section{Street Soccer as an Educational Tool for Social Transformation in Pedro Aguirre Cerda District: the PAC Gol Organization} SUMMARY

The article analyzes how the street soccer methodology strengthens identity building amongst young people who participate in the Pac Gol community organization, located in Pedro Aguirre Cerda District, based on a community education model that confronts the reproduction of sexual, racial, and class stereotypes that are present in the formal education model and in elements of traditional soccer. A qualitative methodology was used, addressing the full sample, combining semi-structured interviews with participant

Chileno. Sociólogo, Universidad Alberto Hurtado, Colectivo Mestizaje- Fútbol y resistencia. E-mail: jigutie.92@gmail.com 
El fútbol callejero como herramienta educativa de transformación social en la comuna de Pedro Aguirre Cerda: organización Pac Gol / Gutiérrez

observation. The project succeeded in identifying members' linkages with the organization - expressed in terms of affective bonds of reciprocal trust between stakeholders, the appropriation of space, and subjective wellbeing amongst members - as well as evidence on changes in schemata, norms, and attitudes amongst participating players, regarding values linked to social inclusion and non-discrimination.

Keywords: identity, popular education, street soccer, social capital

\title{
O futebol de rua como ferramenta educativa de transformação social no município de Pedro Aguirre Cerda: organização Pac Gol
}

RESUMO

\begin{abstract}
$\mathrm{O}$ artigo analisa como a metodologia do futebol de rua fortalece a construção da identidade dos jovens participantes da organização comunitária Pac Gol, localizada no município de Pedro Aguirre Cerda, desde um modelo de educação popular que confronta a reprodução de estereótipos sexuais, raciais e de classe presente no modelo de educação formal e em dimensões do futebol tradicional. Utiliza-se uma metodologia qualitativa e de corte transversal, que combina a realização de entrevistas semiestruturadas com observação participante. Conseguiu-se, por um lado, identificar o arraigo de seus membros quanto à organização - expressado tanto em laços afetivos de confiança recíproca entre seus atores como na apropriação territorial e no bem-estar subjetivo dos indivíduos- e, por outro, a evidência sobre mudanças de pautas, normas e atitudes nos jogadores participantes, em torno a valores relativos à inclusão social e a não discriminação.
\end{abstract}

Palavras-chave: identidade, educação popular, futebol de rua, capital social

Fecha de Aceptación: 14-08-2019

\section{Introducción}

El fútbol, en tanto práctica psicosocial, es una manifestación sociocultural que traspasa fronteras tecnológicas, económicas y políticas, convirtiéndose en multitudinario objeto de interés, pasión y práctica. Se ha transformado en un hecho social total de carácter masivo, que antecede a la globalización y, a la vez, logra insertarse, siendo parte de ella, como un elemento más de su contexto y sus fenómenos (Aguirre \& Pinto, 2006; Carrión, 2016; Matus, Villanova \& Puig, 2016). 
No obstante, la historia oficial y un gran número de intelectuales lo han menospreciado, asociándolo a una mera trivialidad interesante cargada de historia, pasando por alto diferentes dimensiones socioculturales que atraviesa (Carrión, 2016; Vélez, 2016; Cruz, 1998). Para Carrión (2016), las investigaciones sobre este deporte pasan por alto la relación que guarda con construcciones sociales en torno a territorialidad, mientras que, según Aguirre \& Pinto (2006), se dejan a un lado sus alcances como actividad asociativa.

Otros investigadores, como Moreira (2016) y Fernández (2016), sostienen que las ciencias sociales han minimizado la dimensión corpórea del fútbol, ligada a las relaciones de dominación en torno al género, raza y clase que esta práctica esconde (Durán \& Jiménez, 2006). Esta omisión se debe a que, durante el siglo XX, la sociología y antropología aún consideraban descabellado y fuera de lugar asociar al fútbol con producción de conocimiento, puesto que las universidades estaban demasiado ancladas bajo el alero epistemológico positivista-cartesiano, lo que se tradujo en importantes limitantes al momento de investigar la vida social (Vélez, 2016).

Cabe mencionar que la dimensión que se fue construyendo durante el siglo pasado en torno al fútbol, moderna, global, mercantil, de espectáculo-mercancía y sumamente publicitada por los medios, coexiste complementaria y estrechamente con aquella que surgió primero en el tiempo: la original que nació en la ruralidad y en suburbios de las ciudades europeas (Cruz, 1998).

En Chile, la popularidad del fútbol también es un hecho tangible (Cruz, 1998; Carrión, 2016; Campos \& De Ferrari, 2017), país que, desde una perspectiva psicosocial, presenta rasgos individualistas (Páez, 2003), asociados a una sociedad consumista, adultocentrista (Duarte, 2005) y patriarcal (Duarte, 2005; Páez, 2003), en la que el valor de lo colectivo, asociativo y solidario se encuentra en crisis (Urzúa, Alvarez \& Gaete, 2014, p. 198). Consecuentemente, el crecimiento económico o la estabilidad en Chile no se condice en absoluto con un desarrollo equitativo; todo lo contrario, ha generado profunda segregación (Vásquez, 2012). 
El fútbol callejero como herramienta educativa de transformación social en la comuna de Pedro Aguirre Cerda: organización Pac Gol / Gutiérrez

En un escenario de profunda desigualdad, tal como lo es Chile, los jóvenes resienten y absorben con mayor fuerza estas pautas culturales, potenciadas también cuando se trata de sectores populares. En estos estratos sociales, que han sido bombardeados por publicidad con fuertes cargas simbólicas hipersexualizadas y que aluden a un imaginario exitista desde lo económico (Duarte, 2000; Gutiérrez, 2005), el fútbol emerge como reflejo de sus sociedades, plasmando en cierto grado las mismas paradojas que se extrapolan a distintas esferas de la vida social (Cruz, 1998; Carrión 2016; Fernández \& Constela, 2016; Campos \& De Ferrari, 2017), por lo que profundizar en los imaginarios y la apropiación cultural que esta práctica deportiva otorga, proporciona pistas de lo que ha sido nuestro desarrollo social popular y masivo (Cruz, 1998).

Partiendo de estas miradas - desarrolladas fuera del ámbito del fútbol-, que lo ven como un deporte mercantil, masificado y globalizado, nuestra investigación busca comprender la importancia de abordar las organizaciones comunitarias juveniles ancladas al fútbol y su versión callejera a la hora de comprender la potencialidad que estas tienen para fortalecer identidades comunitarias y modelos de educación alternativa. En consecuencia, la presente investigación se plantea: ¿de qué manera el fútbol callejero contribuye a fortalecer la identidad comunitaria de los jóvenes integrantes de PAC Gol, sobre la base de un modelo de educación popular, en la comuna de Pedro Aguirre Cerda en un contexto de vulnerabilidad? Y según esta, ¿cómo se construye la identidad en la comunidad a través de la práctica del fútbol? Por último, ¿de qué manera esta actividad educativo-deportiva proporciona valores de integración social y no discriminación a sus jóvenes?

\section{Fútbol e identidad: revisión histórica de su desarrollo en Latinoamérica y Chile, y su anclaje en lo popular}

Antes de profundizar en las significancias de la metodología del fútbol callejero (MFC) y su nexo con la identidad, el capital social y la educación popular, es necesario comprender los antecedentes generales del fútbol como práctica deportiva en nuestro con- 
tinente. Su arribo no se produjo como mera práctica deportiva, sino que vino acompañado de la imagen de modernidad e integración social, vinculado a identidades de pertenencia territorial y humana, a través de procesos de socialización traducidos en el encuentro de diversos estratos sociales en relativas condiciones de igualdad y con potenciales miras a la emancipación (Carrión, 2016; Cruz, 1998). Su influencia se torna cultural en la medida en que la apropiación de las masas populares llegó a convertir dicha práctica en una pasión de alcance transnacional (Cruz, 1998). En un principio, los clubes constituyeron elementos importantes en la difusión de ámbitos identitarios de base urbana, asimilables a organizaciones sindicales o vecinales. El fenómeno futbolero, además, consagró espacios simbólicos que pueden ser expresión y construcción de identidades clasistas, raciales, masculinizadas y nacionales, considerando que el valor competitivo penetró la esfera de lo popular, más vinculada con el fortalecimiento de ciertas identidades en busca de reconocimiento en la sociedad.

A raíz del establecimiento institucional de la Federación Internacional de Fútbol profesional (FIFA), se propulsaron procesos de mercantilización no exentos de generar desigualdades espaciales y territoriales que tienden a extrapolarse en el nivel local. Aparenta ser democrático, sin embargo, y aun considerando su escala planetaria, es altamente excluyente, al situar a Europa como eje central del imaginario moderno (Carrión, 2016). Estas dimensiones, que también se pueden observar en las lógicas barriales en torno al fútbol, han sido escasamente abordadas por la academia. Lo mismo ocurre en lo referido a las relaciones desiguales en torno al género (Vélez, 2016; Moreira; 2016) o el racismo (Durán \& Jiménez, 2006).

Desde una perspectiva de género, diversas investigaciones han develado pautas culturales asociadas a la práctica del fútbol en el continente latinoamericano: el lenguaje misógino, la cosificación hacia las mujeres, la discriminación y banalización por aquellas mujeres que presentan un interés en practicar o seguir este deporte (Vélez, 2016; Fernández \& Vergara, 2016; Moreira, 2016). Todo 
El fútbol callejero como herramienta educativa de transformación social en la comuna de Pedro Aguirre Cerda: organización Pac Gol / Gutiérrez

esto conlleva al fortalecimiento de estereotipos culturales en torno a la femineidad y masculinidad (Fernández \& Vergara, 2016), $\mathrm{y}$ al fortalecimiento de la división sexual de roles bajo la matriz público-privada, en la que el hombre se consolida en el primer espacio, en desmedro de la mujer, relegada al segundo (Vélez, 2016; Butler, 1990).

El fútbol se conforma como parte de la sociedad (Campos \& De Ferrari, 2017), ya que "condensa simbólicamente las nuevas características que la modernización imprime en éstas” (Cruz, 1998, p. 11), mientras que los voraces flujos mercantiles tensionan lo esencial del fútbol sudamericano: ser una pasión, un sentimiento.

\section{Coordenadas para un "estado del arte" sobre estudios de fútbol comunitario en Chile}

Chile no se exime de la influencia sociocultural descrita (Campos \& De Ferrari, 2017). Considerando la extensión popular histórica del fútbol, se han vislumbrado procesos de difusión y apropiación de la actividad, insertándose como instrumentos que fortalecen la educación popular (Cruz, 1998).

A continuación, se presentan esbozos sobre estudios e investigaciones realizadas en Chile en relación con aquel ámbito menos publicitado por los medios de comunicación: el fútbol comunitario, heredero de los orígenes de este deporte en cuanto práctica de asociatividad, situada en el mundo de la vida popular, coexistente junto a esta visión de mayor visibilidad e impacto en el mundo globalizado. Es expresión de sociabilidad en el ámbito del espacio urbano (barrios y canchas), de carácter voluntario y capaz de aunar significados, experiencias y, a fin de cuentas, identidades compartidas (Aguirre \& Pinto, 2006).

Algunos investigadores concuerdan que en Chile, el desarrollo de estudios sobre organizaciones deportivas es bastante limitado. Empero, se concentran en lo referente a organizaciones deportivas voluntarias, destacando varias de sus características, tales como "fomentar el deporte, (...) la ética y la solidaridad (...), brindar 
oportunidades de educación no formal y fomentar el liderazgo de jóvenes y su participación en la sociedad" (Matus, Vilanova \& Puig, 2016, p. 413). En esta línea, consideran que toda organización enfocada en el deporte y la educación, sea estatal, privada o comunitaria, debe fortalecer sus lazos de cooperación con otras entidades, con la finalidad de consolidar modelos integrativos desde una óptica sociocultural.

Cabrera (2013), por su parte, trabaja sobre la relación entre el rol del Estado y el funcionamiento de las organizaciones comunitarias. Estas, cuando están fortalecidas, crean mecanismos que generan espacios de autonomía y empoderamiento a través de la toma de decisiones. Así, fortalecen la identidad de las personas en cuestión con su comunidad y de la misma comunidad en sí, contribuyendo a mejorar la calidad de vida.

Por tanto, los planos psicológicos y socioculturales serán vitales a la hora de hablar de transformación social. La educación popular representa una piedra angular para dicho cambio, al entenderse como un cambio de conciencias desde abajo (Matus et al., 2013).

Algunos autores discuten acerca de las posibilidades que tendría el fútbol, en tanto práctica psicosocial, de convertirse en proceso de intervención comunitaria. Dicha práctica, considerada como acción social organizada, contribuye al desarrollo de la comunidad. La noción de "desarrollo comunitario" que manejan Urzúa, Alvares y Gaete (2014) se refiere a la interrelación existente entre el desarrollo individual, grupal y de sociedad, en términos ecológicos y desde la acción y participación comunitaria. Emerge cuando la comunidad se empodera y adquiere autonomía ante sus contingencias desde la organización de sus actores en pos de buscar soluciones, potenciando sus recursos y habilidades.

Estos investigadores se focalizaron en los aportes realizados por la institución "Fútbol Más" (perteneciente al Ministerio del Interior), para extraer relatos de jóvenes, adultos y apoderados, con el fin de conocer detalles sobre sus experiencias y significados. Sin embargo, esta iniciativa no logró conformar estrategias sólidas que 
El fútbol callejero como herramienta educativa de transformación social en la comuna de Pedro Aguirre Cerda: organización Pac Gol / Gutiérrez

incentivaran la conformación de capital social. Por ello, se torna relevante indagar sobre actores u organizaciones que gozan de posicionamientos históricos y políticos familiares en sus barrios, que facilitan construcciones y procesos comunicativos (Urzúa et al., 2014). Existe, efectivamente, una necesidad, desde las ciencias sociales y políticas, por analizar al fútbol desde su potencial como proceso de intervención, superando su mera asociación al manejo del tiempo libre y el ocio, y refiriéndose a sujetos categorizados como "vulnerables".

Todas estas investigaciones sobre el fútbol popular evidencian cómo las organizaciones comunitarias que lo abordan desde su dimensión local, surgen contraponiéndose al fútbol comercial y privatizador en Chile y su consiguiente anclaje a pautas culturales predominantes.

Por último, cabe mencionar la relevancia de la organización comunitaria Chigol, ubicada en la población El Montijo de la comuna de Cerro Navia, que remite directamente al origen y expansión de la Metodología de Fútbol Callejera (MFC), que busca potenciar métodos que promuevan y fortalezcan procesos de desarrollo identitario juvenil en torno a valores de autonomía y liderazgo.

\section{Identidad y género como construcciones discursivas}

En la modernidad tardía, la identidad de los sujetos está construida de múltiples maneras, a través de discursos, prácticas y posiciones diferentes, a menudo cruzadas y antagónicas. De allí que se presente de forma fragmentada y múltiple, cuando en ella convergen nuevas formas de movilización política que ponen en entredicho - y al mismo tiempo reconfiguran - los viejos conceptos de "clase", "género", "sexualidad", "raza", "etnia" o "nacionalidad". Pero este proceso actúa en doble sentido, pues no solo repercute en el andamiaje teórico de las ciencias sociales, sino también en el mismo concepto de "identidad".

Por lo tanto, la identidad es una construcción, un proceso siempre inacabado (Hall \& Dugay, 2003). Según esta definición, la identi- 
dad es una construcción cuyo proceso no es estable sino dinámico y contingente. Su transformación va impulsada por las prácticas discursivas. No obstante, dichos autores aclaran que se constituyen dentro de la representación y no fuera de ella, dentro del discurso y no fuera de él. En consecuencia, las identidades son temporarias a posiciones subjetivas que construyen las prácticas discursivas mencionadas. Son producto de una articulación del sujeto en el flujo del discurso.

Uno de esos discursos fundacionales de la modernidad temprana fue la conformación del género, mediante explicaciones biologicistas de la naturaleza humana que catapultaron, por un lado, a los hombres al mundo de la política y el trabajo, y, por otro, a las mujeres al mundo de lo privado: la reproducción y crianza de los hijos. Al respecto, Butler (1990) trabajó las transacciones complejas entre el sujeto, el cuerpo y la identidad, para luego relacionarlas dialécticamente con procesos de exclusión y dominación. De manera similar, Scott (1990) sostiene que el género es una construcción social derivada de relaciones de poder. De allí que considera que el lenguaje y el discurso posicionan al sujeto como ente analizable; por lo tanto, bajo la mirada de Scott (1990), el sujeto adquiere existencia social en la medida que se le entiende como objeto de conocimiento y de prácticas discursivas construidas y transformadas a través de la historia.

\section{Identidad y capital social}

El "capital de identidad" es un término inspirando en la sociología de Pierre Bourdieu, pero desarrollado por Côté \& Levine (1997, 2002), quienes lo definen como los recursos y activos que una persona despliega en situaciones que se relacionan con su autodefinición. Parafraseando a Goffman (2001), el capital de identidad sería el trabajo que hacen los actores para generar una impresión de sí mismos en la audiencia. En consecuencia, el capital social y cultural, así como el arsenal de herramientas psicosociales (Giménez, 1997), constituyen recursos empleados por los sujetos para moverse reflexivamente en las dimensiones de lugar, espacio y 
El fútbol callejero como herramienta educativa de transformación social en la comuna de Pedro Aguirre Cerda: organización Pac Gol / Gutiérrez

tiempo (Giddens, 2008), dentro del mundo de modernidad tardía (Hall \& Dugay, 2003).

La teoría de Bourdieu (2000) también proporciona elementos para identificar y tipificar los capitales en los que se basa la dominación en diversos campos sociales (Joignant, 2012), debido a que existe una disputa en la que se correlacionan fuerzas al interior de ellos por el valor según los capitales desplegados, dentro de los cuales se puede identificar un capital en específico, relevante al momento de hablar de identidad colectiva: el "capital social". Este último concepto remite a la capacidad que tienen los individuos para movilizar a su favor el capital de un grupo más o menos institucionalizado y rico en capital. El capital social se conformaría por:

(...) la totalidad de los recursos potenciales o actuales asociados a la posesión de una red duradera de relaciones más o menos institucionalizadas de conocimiento y reconocimiento mutuos. Expresado de otra forma, se trata aquí de la totalidad de recursos basados en la pertenencia a un grupo. (Joignant, 2012, p. 148)

La definición precedente destaca cuatro elementos esenciales que intervienen en su conformación. El primero es la pertenencia a un grupo. El segundo, las relaciones de intercambio material y simbólico. En tercer lugar, su grado de institucionalización y legitimación, y, por último, los recursos que posee dicho grupo (Plascencia, 2005).

A diferencia del capital económico y cultural, relacionado directamente con los individuos, el capital social demanda un grupo o una red duradera de relaciones sociales. En este contexto, Durston (2000) se vale de la dimensión colectiva y grupal de capital social delineada por Bourdieu (2000), Putnam (2002) y Coleman (1990) para definir el capital comunitario como:

El capital social colectivo o comunitario (...) consta de las normas y estructuras que conforman las instituciones de coo- 
peración grupal. Reside no en las relaciones interpersonales diádicas, sino en estos sistemas complejos, en sus estructuras normativas, gestionarias y sancionadoras. Recordemos, al respecto, que las comunidades son mucho más que redes; mucho más, incluso que redes circunscritas (...) La definición clásica de comunidad abarca aspectos de actividad coordinada con cierto propósito común, autogobierno, superestructura cultural, y sentido de identidad. (Durston, 2000, p. 21)

Desde esta perspectiva, toda colectividad portadora de identidad requiere de capital social, no solo para su funcionamiento, sino también para existir socialmente dentro de una dimensión simbólico-cultural (Giménez, 1997). De allí que el capital social o comunitario (Durston, 2000) pueda interpretarse como un mecanismo que contribuye a configurar no solo la identidad grupal o colectiva mediante la movilización de los recursos y las redes, sino también como mecanismo de diferenciación social y reproducción de las clases (Bourdieu, 2000).

Como se ha visto, la idea de pertenencia grupal entraña varias interpretaciones articuladas entre sí. La primera es que la identidades son dinámicas y se sitúan en el discurso y la representación (Hall \& Dugay, 2003), originados a partir de una experiencia común en un conjunto de informaciones, creencias u opiniones. La segunda es que la identidad no solo se configura por oposición sino también por asimilación, como un juego de poder dentro de determinado campo (Bourdieu, 2000; Butler, 1990; Scott, 1990). Tercero: la configuración, tanto de la identidad individual como colectiva, comprende una relación dialéctica, que reposa en los criterios de distinguibilidad propios de procesos de pertenencia, que a su vez dotan a los individuos de una internalización de representaciones sociales (Giménez, 1997). Por último, el rol del capital social para incubar pautas y valores en los individuos mediante interacciones asociativas tiene lugar dentro de la trayectoria de ellos, quienes están inmersos en una trama colectiva o comunitaria (Durston, 2000). 
El fútbol callejero como herramienta educativa de transformación social en la comuna de Pedro Aguirre Cerda: organización Pac Gol / Gutiérrez

\section{Educación popular como una alternativa contra los valores individualistas y estereotipos sexuales, raciales y de clase presentes en la educación formal}

La educación formal se caracteriza por una estructura vertical, autoritaria, que no entiende el proceso educativo como un proceso que permita la integración significativa de valores en los niños y jóvenes, sino que apunta a la mera repetición de contenidos desligados de las motivaciones de estos actores (Freire, 1997; Martínez, 2015; Páez, 2003). Por otro lado, el colegio, institución acogedora de dicho modelo, se ha constituido socialmente como un espacio de desarrollo, en circunstancias que, muchas veces, representa un ámbito de violencia simbólica y reproducción de estereotipos sexuales, raciales y de clase (Preciado, 2016).

Opuesta a este modelo de educación formal, que surgió en el siglo $\mathrm{XX}$, la educación comunitaria responde a una propuesta filosófica alternativa, que deja al descubierto las limitaciones de la educación formal, tales como la falta de articulación entre pedagogía, procesos psicológicos, cognitivos y sociales de niños y jóvenes (Freire, 1996).

Esta nueva filosofía, que da sustento a una propuesta de educación alternativa y liberadora, ha sido ampliamente debatida hasta el punto de atribuir a Freire cosas que no ha dicho o bien que sostuvo, pero que se descontextualizan (Martínez, 2015). En esta línea, existen autores que niegan las contribuciones y la autenticidad de sus aportes (Pavia, 1982; Pérez, 2001), pero otros, como Martínez (2015), opinan que Freire efectivamente realizó importantes contribuciones en materia humanista y relativa a los movimientos sociales de Latinoamérica, además de crear un constructo filosófico sobre educación, que constituyó una teoría del conocimiento articuladora de modelos y prácticas de aprendizaje concretos, nunca desligados de las coyunturas sociales o políticas.

El pensamiento de Freire recibió influencias de autores como K. Popper y Pierre Furter, al poner en entredicho la estructura tradicional del currículo pedagógico, en pos de que este se relacione 
con el sujeto y la generación de perspectivas críticas respecto del entorno (Martínez, 2015).

Freire (1968) parte de la base que la globalización implanta mecanismos de dominación en una estructura social en la que opera la razón instrumentalizada, responsable de producir una violencia simbólica en diversos espacios de la vida social, potenciada por los medios masivos. Esta pedagogía predominante se ajusta a la economía global de mercado, que busca perpetuar la existencia de sujetos y colectivos dominantes privilegiados, en desmedro de otros oprimidos. Desde esta perspectiva, la educación dominante se erige como "un obstáculo epistemológico que impide una vinculación pedagógica con el desarrollo social-comunitario" (Pérez \& Sánchez, 2005, p. 319).

Ante este obstáculo, Freire (1996) plantea una pedagogía de la liberación, que contempla algunos puntos medulares que permiten comprender su filosofía sobre una educación anclada en lo popular. Estos postulados pueden resumirse de la siguiente manera:

1. La pedagogía debe fomentar el pensamiento crítico de los sujetos, de modo que estos desarrollen un crecimiento intelectual ligado a un ejercicio de reconocimiento profundo respecto al entorno que los rodea. Esta conciencia formada se corresponde con la conciencia histórica, apostando por visibilizar las relaciones de poder existentes. Este proceso educativo se comprende como un ejercicio permanente, que se extrapola a todo momento y ámbito de la vida social de los individuos, problematizando los sucesos.

2. La libertad resulta un componente principal para fortalecer ciudadanos autónomos. Empero, ésta tiene sus límites en la medida de no pasar por alto aspectos éticos mínimos, relativos al respeto y la aceptación plena de los demás, dejando de lado cualquier prejuicio, ya sea de tipo sexual, clasista o étnico, comprendiendo históricamente que la alteridad ha sostenido los procesos de cosificación humana. 
El fútbol callejero como herramienta educativa de transformación social en la comuna de Pedro Aguirre Cerda: organización Pac Gol / Gutiérrez

3. Este modelo entiende la democracia no desde una óptica que se centra en los intereses de una mayoría de votantes, sino centrada en propiciar espacios de diálogo abiertos, que busquen el consenso entre actores, de manera que todos los participantes reflexionen en torno al bien común como primordial horizonte.

4. La pedagogía educativa promueve relaciones horizontales entre jóvenes y sus docentes. El docente representa una figura de apoyo para los estudiantes y debe enfocar un trabajo personalizado sobre ellos.

5. Las actividades desarrolladas por los estudiantes tienden a ser elegidas por ellos mismos, potenciando su creatividad, explotando sus motivaciones, su capacidad de liderazgo y autonomía, y el trabajo en equipo.

6. El encuentro entre las conciencias individuales con las colectivas pueden conllevar a una acción comunitaria de transformación social.

La propuesta filosófica de Freire, al propiciar espacios de diálogo, sinergia el trabajo individual con el colectivo y, con ello, genera las condiciones para la emergencia de capital social comunitario (Durston, 2000). También puede abordarse desde un enfoque psicosocial, pues fortalece la identidad de los sujetos desde una mayor intensidad afectiva (Páez, 2003), y también desde una mayor armonía entre actitudes y conductas, como una alternativa a ese modelo de educación formal denunciado por Freire (1996), que consolida identidades asociadas a valores materialistas y a pautas, hábitos, normas y conductas verticales y autoritarias que tienen lugar a nivel macrosocial (Páez, 2003).

\section{Fútbol callejero y educación popular}

Como actividad participativa-educativa-deportiva y cultural, la metodología de fútbol callejero (MFC) armoniza la propuesta freiriana $(1968,1997)$ sobre la necesidad de vincular tanto los procesos de enseñanza con el desarrollo social-comunitario (Pé- 
rez y Sánchez, 2005), como con los valores que promueven la conformación de capital social (Bourdieu, 2000; Putnam, 2002) en el ámbito de las comunidades (Durston, 2000). Al respecto, la MFC se constituye a partir de un conjunto de organizaciones sociales que, a través del fútbol callejero, comparten la misión de construir ciudadanía, defender los derechos humanos y de la naturaleza, abogar por la justicia, promover una sociedad inclusiva y reconocer la diversidad cultural, étnico-racial y de opciones.

La MFC en todo el mundo se presenta como una forma de conceptualizar y entender el fútbol en tanto estrategia para crear y acompañar procesos de aprendizaje e inclusión social, recuperar los valores humanos, impulsar el desarrollo de liderazgos y generar procesos comunitarios solidarios de transformación. De allí que las organizaciones promuevan la práctica del fútbol callejero apuntando al desarrollo de los jóvenes en pos de la transformación social. Desde la metodología de los tres tiempos, los jóvenes consensúan las normas de juego con base en respeto y compañerismo y no discriminación, sin la necesidad de un árbitro clásico, sino acompañados por monitores que los guían en sus actividades.

\section{La metodología de fútbol callejero y la posibilidad de generar capital social}

La investigación de Valenzuela (2012) sostiene que los indicadores clásicos de capital social, tales como la confianza interpersonal y la confianza social, pueden anclarse coherentemente y complementariamente a elementos tales como la solidaridad de pares, los procesos de participación, las oportunidades de participación activa y creencias de eficacia política, en pos de reajustar y articular un concepto más robusto y asociado a jóvenes. Su modelo teórico resultó ser una reconstrucción del concepto de capital social, y se caracteriza por recibir una importante influencia de Durston (2000).

Por otro lado, Aguirre \& Pinto (2006) indagaron percepciones de actores sociales de un club de fútbol amateur, con la pretensión 
El fútbol callejero como herramienta educativa de transformación social en la comuna de Pedro Aguirre Cerda: organización Pac Gol / Gutiérrez

de recopilar información sobre relaciones interpersonales, significados y redes asociativas, en un trabajo cualitativo basado en la teoría de los campos de Bourdieu (2000). A raíz de esto, se desprende el uso de conceptos tales como "campo", "capital social", "redes sociales, "asociatividad", "capital simbólico, "capital cultural" y "habitus", relacionados con la actividad futbolera. Aguirre \& Pinto (2006) también integran a su modelo teórico los aportes de Durston (2000), quien realza el valor de la socialización temprana en la integración del capital social en la personalidad y los proyectos de vida.

El capital social es entendido como "un contenido de confianza y cooperación de las relaciones sociales" (Pinto \& Aguirre, 2006, p. 9), que permite el despliegue de las relaciones entre personas, desde el cual puede potencialmente producirse un capital social comunitario. De esta manera, el espacio geográfico se torna susceptible de coincidir con el capital social. Para que esto ocurra, es necesaria la existencia de experiencias precursoras (Durston, 2000) que constituyan la base concreta de dicho capital:

Memoria social e histórica; identidad y etnicidad; religiosidad compartida; vecindad y cercanía geográfica; amistad y compañerismo; relaciones de parentesco; principios y prácticas de reciprocidad. Cada una de estas experiencias propicia el surgimiento de la confianza y la cooperación como elementos articuladores de capital social (...) por lo tanto existe una estrecha relación entre las pautas sociales y culturales a la hora de hablar de producción de capital social comunitario. (Aguirre, 2006, p. 9-10)

\section{Metodología}

El tipo de investigación fue de corte transversal. Se sitúa en un momento específico de tiempo y de nivel explicativo, ya que busca relacionar variables y conceptos que sean importantes para el estudio en cuestión (Hernández, Fernández \& Baptista, 2010).

La muestra y sus criterios está constituida por miembros de PAC Gol: cuatro jugadores de entre 14 y 17 años que llevaran por lo 
menos 6 meses en la organización y tres monitores mayores de 18 años. Se suma el documento de principios internacionales de la MFC y fuentes audiovisuales secundarias, en el que aparecían entrevistas a monitores/ex jugadores, a una apoderada y su director.

La recolección de datos fue a través de 7 entrevistas semiestructuradas y el desarrollo de observación participante. La utilización del primer método se justifica, dado que ofrece un grado parcial de estructuración bajo una pauta o guía temática, pero, siendo esta flexible, permite al investigador introducir con libertad nuevas preguntas, con el fin de precisar o profundizar en los conceptos anhelados (Hernández, 2010). Los jóvenes entrevistados tenían entre 14 y 16 años, mientras que los monitores fluctuaban entre los 19 y 26 años. Las entrevistas fueron realizadas entre el 3 y el 21 de noviembre de 2017. La antigüedad de la que databan las trayectorias de los jóvenes en Pac Gol fluctuaban entre 6 meses y 3 años.

La observación participante en terreno fue en 2 de las 5 poblaciones abarcadas por la organización, La Nueva Independencia y La Victoria, a lo largo de cinco semanas, específicamente entre los días 18 de octubre y 25 de noviembre, considerando que esta implica un involucramiento activo y profundo con los ambientes y contextos sociales que se observan durante el estudio (Hernández, 2010). El involucramiento no es absoluto, pero si hay una penetración en la articulación de actividades desarrolladas, que se expresó en jugar con los muchachos en algunos entrenamientos, sumado a ayudar a los monitores con las actividades y el apoyo técnico al equipo en la liga de los sábados. En el primer territorio se observaron los entrenamientos de los días miércoles y viernes, mientras que el segundo fue visitado cuatro veces para observar el desarrollo de la liga "La Marina", que se juega cada sábado.

Respecto del plan de análisis, el tratamiento de los datos se realizó a partir de un análisis de contenido cualitativo, con categorías a priori (Echeverría, 2005), que buscaba conocer significados, temáticas y sentires que se encuentren en el entramado discursivo de los sujetos entrevistados. Su utilización se justifica ante la pre- 
El fútbol callejero como herramienta educativa de transformación social en la comuna de Pedro Aguirre Cerda: organización Pac Gol / Gutiérrez

sencia de un objetivo y pregunta acotada y que se cuente con antecedentes teóricos suficientes sobre el tema. Esto se corresponde con utilizar cierta estructuración por parte del investigador, cuya predefinición asume un trabajo con mayor fragmentación en la mirada de la información y, por tanto, una delimitación relativa de los puntos de vista de los sujetos.

Para responder la pregunta de investigación, la información recopilada se analizó al interior de dos categorías, con las características ya mencionadas. Luego, en segunda instancia, se realizó un análisis de cada categoría en específico, articulando un texto basado en las experiencias subjetivas del entrevistado, guiado por la creación de tópicos.

\section{Aproximación al objeto de estudio}

La comuna de Pedro Aguirre Cerda, donde se encuentra la organización PAC Gol, se sitúa en el sector centro-sur del Gran Santiago, limitando al norte con la comuna de Estación Central y Santiago, al este con la comuna de San Miguel, al oeste con la comuna de Cerrillos y al sur con la comuna de Lo Espejo. La última estimación de población del año 2015 revela una cantidad aproximada de 122 mil habitantes y una tasa de pobreza por ingresos de $8 \%$ (CASEN, 2015).

La definición y misión de esta organización comunitaria utiliza la MFC, el arte muralista, batucadas y la cultura callejera como herramientas educativas en pos de la transformación social. Desde este fútbol diferente a expresiones culturales y artísticas se puede educar en un proceso constante y horizontal, alejado de las lógicas formales y verticales, para mejorar la calidad de vida de las poblaciones y la comunidad. La comunicación y la participación son piedras angulares para dichos objetivos.

La organización da cuenta en su sitio web sobre sus inicios en 2007, a raíz de los esfuerzos mancomunados de jóvenes provenientes de la misma población La Victoria, quienes instauraron la sede localmente, buscando conjugar un proyecto educativo ancla- 
do, en primera instancia, en la práctica futbolera desde un ángulo diferente al tradicional. Cabe mencionar que dicha población se convirtió en la primera toma de terreno organizada de Chile y Latinoamérica (Lemuñir, 2017), reforzando un perfil social en la comuna, producto de un proceso histórico compartido de reivindicaciones organizadas de los sectores de trabajadores, que en la actualidad da cuenta de una gran capacidad de organización y participación; no obstante, sus poblaciones en general presentan importantes focos de vulnerabilidad, propios de una comuna con altos índices de pobreza y bajos recursos.

Los miembros de esta organización comunitaria lo componen sus jugadores (as) y monitores (as), sumado a vecinos y apoderados. Desde sus inicios, surgen iniciales financiamientos autogestionados a través de fiestas, rifas y completadas. La construcción social para este colectivo se centra en los valores ya descritos de MFC y su potencial transmisión generacional. Participan en ella habitantes de la Población La Victoria desde 2008; San Joaquín y Nueva Independencia desde 2010; Nueva Villa Sur desde 2011, y Población Dávila desde 2013, y sus actividades de fútbol callejero permiten la participación de casi 300 niños y jóvenes.

\section{Análisis y discusión de resultados}

Para exponer los resultados se emplearon dos categorías principales. La primera es "capital sociocomunitario en la organización juvenil y desafíos futuros", mientras que la segunda se refiere a la "evolución en las pautas, normas y conductas de los jugadores en base a valores de inclusión y no discriminación en un contexto vulnerable". Dichas categorías se explican a continuación:

\section{a. Capital sociocomunitario en la organización juvenil y desafíos futuros}

Dentro de esta categoría, los hallazgos están asociados a discursos extraídos de diferentes entrevistados miembros de PAC Gol acerca de su visión del capital social comunitario y la conformación de 
El fútbol callejero como herramienta educativa de transformación social en la comuna de Pedro Aguirre Cerda: organización Pac Gol / Gutiérrez

redes de confianza y solidaridad, que promueven la participación política, es decir, en las que cada sujeto tenga las posibilidades y derechos de aportar a su comunidad de manera compenetrada, crítica y constructiva. Al respecto, la primera reflexión que se desprende se relaciona con el nexo de los entrenamientos semanales y la participación del equipo en la liga "La Marina", que tiene lugar los sábados. Se entiende, desde lo observado, que la puesta en marcha del habitus futbolero en la esfera barrial-comunitaria saca a relucir un carácter asociativo y de interacción social. Estas construcciones presenciales y horizontales constituyen potencialmente un desarrollo en el capital social para la comunidad y los mismos individuos que la componen.

Los testimonios de los miembros, recogidos durante las entrevistas, develan la existencia de una vinculación de sus prácticas y discursos con la filosofía de PAC Gol. Esta filosofía, en ellos, no se refiere al mero hecho de jugar al fútbol, sino que además incorpora su esfera analítica y política respecto a la actividad, relacionada directamente con la toma de decisiones, el intercambio de argumentos y la reflexividad grupal e individual, posterior a finalizar las jornadas de entrenamientos o partidos, en pos de llegar a consensos que estén a la par con objetivos comunes que contribuyan al desarrollo colectivo de todos sus miembros. Esta forma de ser y actuar se manifiesta en prácticas de diálogo y exposición dentro y fuera de la organización, tal como se refleja en el siguiente testimonio del director de PAC Gol:

(...) Pero después, producto de que se van generando procesos de diálogo, en donde ya los monitores tienen esa capacidad, que también es mágica, de generar estos procesos facilitadores de educación y comunicación, es donde ellos comienza a sentir, y donde ya hay generaciones que tienen la metodología y la forma más internalizada, es más fácil.

Desde la línea de la internalización de pautas y costumbres, se observa que la identidad individual de los jóvenes asumirá discursos relacionados a un sentido de permanencia por PAC Gol, reforza- 
dos con mayor fuerza en las actividades en las que jueguen como equipo contra otras colectividades. Destaca, por tanto, la existencia de un anclaje funcional entre los entrenamientos de los días miércoles y viernes en los distintos territorios de intervención con la liga del sábado "La Marina". En dicha instancia competitiva, PAC Gol va a participar como un equipo, enfrentando a otros clubes o asociaciones de la comuna.

Las entrevistas a los miembros de esta organización también revelaron que la creatividad y la libertad están presentes en el desarrollo de los entrenamientos, en tanto ciertos niveles de autonomía a la hora de reglamentar el juego. Así se potencia la asociatividad, en la cual las individualidades interactúan bajo pautas y valores que encasillan requisitos mínimos de conductas esperadas relacionadas con el respeto, y en la que se yerguen las premisas de articular cualquier asunto, sea conflictivo o no, desde la horizontalidad y el ejercicio reflexivo, tal como se refleja en el siguiente testimonio:

(...) Ellos después evalúan qué cosas lograron y que cosas no, y decir, si lo cumplí, la regla que pusimos, admito que lo hice, o admito que no, admito que lo hice bien o que jugué mal (...) que dije un garabato, que estuvo mal, pido disculpas, no merezco el punto, mi equipo no lo merece porque yo no cumplí. Ellos son lo más importante, ellos son todo esto (...) yo siento que acá ellos pueden ser ellos mismos. (Monitora, adulta joven, comunicación personal, 19 de noviembre de 2017)

Es más, desde aportes psicosociales ligados con el ejercicio pedagógico, se asume que las actividades cooperativas y elegidas voluntariamente refuerzan más la motivación intrínseca (Páez, 2003), con lo que concuerdan Aguirre y Pinto (2006) en sus estudios sobre fútbol comunitario.

Esta articulación de actividades, recién mencionada, genera una identificación colectiva para con el grupo-equipo, al estar por ejemplo vistiendo una indumentaria igual para todos y con insignias estampadas, organizar los botellones de agua para los partidos, charlas técnicas y todo lo que implica un aproximamiento 
El fútbol callejero como herramienta educativa de transformación social en la comuna de Pedro Aguirre Cerda: organización Pac Gol / Gutiérrez

en vivo de la experiencia competitiva del fútbol, mientras que a los monitores les corresponde la labor de "DT" (director técnico). Muchos niños "tienen el deseo de ser futbolistas" o, simplemente, vivir estas instancias competitivas como equipo y empaparse de lo que entrega dicha experiencia, aprendiendo de las derrotas y victorias. Los monitores por tanto, se esfuerzan en entrelazar las actividades descritas con experiencias de bienestar respecto de lo que es la participación, tanto desde los monitores como desde los jugadores. Se comprende, a raíz de esto, que se constituya un arraigo al grupo y también a los espacios en que este se desarrolla, cargados de símbolos para los sujetos, considerando que el "campo" brinda importancia a la dimensión histórica.

Para comprender esta sincronía en el funcionamiento semanal de la organización es vital captar la relación particular entre sus actores. Por un lado, se percibe una buena y fluida relación entre los coordinadores, quienes buscan articular de mejor manera la limitación de recursos materiales con los tiempos disponibles en la semana para lograr levantar las actividades y entrenamientos. Por otro, se observan relaciones de confianza y afecto recíproco entre jugadores y monitores, pero no dejando de existir por dicha razón el respeto entre los actores, procurando que no se den abusos de confianza:

(...) Sí, buena, porque tenemos confianza (...) sobre todo con el Camilo, sobre todo con él. Sí, hay harta amabilidad (...) como decirlo, son como (...) Yo confío en ellos y ellos confían en mí. (Jugador adolecente, comunicación personal, 12 de noviembre de 2017)

La imagen del coordinador busca plasmar una guía educativa, que establezca límites pero también signifique un apoyo moral, afectivo e incluso material para los jóvenes, quienes viven en ambientes vulnerables, tanto en la esfera escolar, barrial como familiar. Son de gran relevancia aquellos casos en que los jóvenes llegan a identificarse fuertemente con algún monitor, vislumbrándolo como ejemplo a seguir: 
(...) Pero el referente obviamente son la Yomara y la Dani. jEllas la llevan! Tú las viste, ellas igual son de carácter fuerte, cachai, pucha, y a los chiquillos les gustaría llegar a ser como la Yomara. La Yomara jugó a la pelota (...) entonces les gustaría po, les gustaría harto. Se fijan en ella, así que bien po. Y la Yomara que lleva tanto tiempo trabajando. (Monitor, adulto joven, comunicación personal, 14 de noviembre de 2017)

Los monitores, por su parte, visibilizan la necesidad de afecto y apoyo que muestran los jóvenes, cuyo espacio lo encuentran en PAC Gol. De esta manera, el fútbol puede ampliarse como una práctica psicosocial no solo de educación, sino también como un espacio de encuentro y contención afectiva.

Ahora bien, sumando los aspectos mencionados, en los que se ha hablado del nexo entre la MFC con la liga "La Marina", el bienestar subjetivo de los sujetos en PAC Gol y la relación de afecto, cooperatividad y asociatividad entre jugadores y monitores, cabe destacar las percepciones y sentires que se expresan en los discursos de sus actores, con énfasis particular en la correspondencia entre la labor que despliega respecto de los objetivos organizacionales y principios del MFC, y los posibles aspectos a mejorar a futuro.

En líneas generales, los miembros, tantos apoderados, el director general, jugadores y monitores, evalúan de manera satisfactoria la labor de la organización, comprobando una correspondencia entre sus principios y objetivos respecto de la vivencia subjetiva de quienes la componen y los avances percibidos:

(...) de un 1 al 10 (...) mmm, siempre nosotros queremos más, entonces yo lo pondría como un 9, podría perfectamente ser un 10, pero yo sé que podemos más. Un 9, un 9 (...) yo sé que podríamos nosotros ser un 10, pero siempre queremos más, por tanto un 9 (...) siempre estamos con proyectos, con cosas, que pueden ser más, entonces un 9. (Monitora, adulto joven, comunicación personal, 19 de noviembre de 2017) 
El fútbol callejero como herramienta educativa de transformación social en la comuna de Pedro Aguirre Cerda: organización Pac Gol / Gutiérrez

Los sutiles aspectos a mejorar, que fueron rescatados desde estos discursos, apuntan mayormente a la esfera material y de recursos. Se vislumbra un asunto interesante respecto de los frutos y la capacidad autónoma, propia de una autogestión seria y comprometida, a pesar de estar en condiciones materiales precarias, tanto en lo barrial como en lo familiar. Los principales desafíos que se recogen son relativos a la necesidad de expansión de instancias educativas como estas, que sean capaces de aunar la actividad deportiva con la participación activa de sus miembros. Los monitores concuerdan con se sienten bastante conformes con las labores realizadas, pero que nunca hay un "techo", siempre hay desafíos por delante.

Los jóvenes, por último, considerando la heterogeneidad natural de sus personalidades, presentan como punto en común el compartir a diario en un entorno en el que se ve fluir mucha droga, violencia, y estigmatización hacia la comunidad migrante. Sin embargo, la característica primordial que se constata se vincula con su pasión por jugar fútbol. La motivación principal de ellos se ancla en la actividad deportiva, recalcando incluso lo positivo de "dejar más de lado la tecnología y participar en el deporte", y usar su tiempo en lo que les apasiona.

\section{b. Evolución en las pautas, normas y conductas de los jugadores en base a valores de inclusión y no discriminación en un contexto vulnerable}

La segunda categoría, por su parte, se refiere a los discursos extraídos por los diversos miembros de la organización, cuyos hallazgos son relativos a la percepción sobre cambios de pautas, valores, conductas y actitudes en sus jóvenes (jugadores), particularmente asociados a temáticas de inclusión y no discriminación de ningún tipo.

En las entrevistas se observó un discurso asociado a la naturalización de la integración a las mujeres, como también con el migrante, superando prejuicios y estereotipos. Los testimonios revelan 
además que los jugadores han asimilado los valores del trabajo en equipo y el respeto entre ellos, y van afianzando sus capacidades de liderazgo constructivo, lejanos a valores individualistas. Desde la psicología social se menciona que el contacto entre grupos, apoyado por las normas colectivas y reconocidas con claridad por sus miembros, en igualdad de estatus y orientado a la cooperación, fortalece los mecanismos en favor de la no discriminación y prejuicios (González \& Fernández, 2003). Al respecto, uno de los coordinadores opina:

(...) nosotros estamos, de una u otra forma, cambiando la realidad de los niños (...) Y a largo o mediano plazo está existiendo un cambio real en el comportamiento de ellos, eso es totalmente comprobable. Yo lo he comprobado. Y lo mismos apoderados, que son los papás de ellos.

Estos cambios son valorados por actores comunitarios en términos positivos, tal como se observa en el siguiente fragmento:

(...) bueno, llegamos a PAC GOL con las niñas porque no se habían podido integrar nunca a otra escuela de fútbol, por ser mujeres; y cuando llegaron ellos aquí, llegó el Jorge y me dijo que necesitaba niños para jugar a la pelota. Le pregunte si se podían integrar niñas, y él me dijo que sí, que ningún problema. Las hice jugar y ahí se quedaron.

Los monitores deben comprender además los límites de su rol, ya que no pueden estar presentes en todos los espacios de los jóvenes. Sin embargo, en la medida en que les proporcionan herramientas que les permitan desarrollar la autonomía, los jóvenes muestran que pueden empoderarse ante su entorno y comprenderlo. En cuanto a la demarcación de límites antes mencionada, se aplican ciertos castigos ante conductas inadecuadas, pero desde una óptica constructiva y conectada con la pasión central de los jugadores. Estos castigos se describen a continuación:

(...) Es un castigo que no duele po, que es un castigo como en el deporte, desde lo deportivo, o sea oye, "vay a poder entrenar 
El fútbol callejero como herramienta educativa de transformación social en la comuna de Pedro Aguirre Cerda: organización Pac Gol / Gutiérrez

pero no poder jugar, entonces ¿para qué entregai? Si no vas a poder jugar en la Liga si te mandaste la mansa embarrada", entonces, es como para decirles "piensa lo que haci", ahí ellos dicen "no, si yo si quiero jugar - la mayoría quieren ser futbolistas-, entonces ¿cómo no voy a jugar? ¿Cómo no me van a ver? ¿Cómo no me voy a probar en un club?, entonces lo tienen muy presente. (Monitor, adulto joven, comunicación personal, 14 de noviembre de 2017)

En el ámbito de la psicología social se comprende que los líderes positivos inspiran admiración sobre sus pupilos, considerando siempre el bien común y no dejando de lado una atención personalizada con ellos (Freire, 1997; Páez, 2003). En síntesis, se entiende que la construcción de identidades juveniles, proceso de por sí vertiginoso (Duarte, 2005), se articula en un entorno altamente vulnerable, lleno de tensiones y ciertas limitantes. Así lo expresa el director de PAG Gol:

(...) yo creo que hay muchos niños que, cuando llegan, llegan con un comportamiento del fútbol tradicional. Ese individualismo, esa poca solidaridad con los compañeros, ese garabato, esa forma que entre más choro soy y más individual soy, soy más bacán, eso es lo que convive en una primera etapa.

Sin perjuicio de lo anterior, se observa que relucen a fin de cuentas cambios positivos en ellos, expresados en un fortalecimiento individual rescatando los valores colectivos. En consecuencia, el fútbol callejero fortalece la identidad colectiva y el capital comunitario, en la medida que intenta reapropiar los espacios comunitarios para los mismos habitantes de las poblaciones, es decir, se busca que la comunidad vuelva a generar sentidos de pertenencia por estos.

Por tejido social roto se refiere a la falta de espacios que propicien un desarrollo de capital sociocultural (Urzúa, Álvarez \& Gaete, 2014). Por lo tanto, este desarrollo comunitario va a tener sus canales o medios no desde salas de clases o instituciones estructu- 
radas, sino que en el espacio público donde, a través de actividades artísticas o deportivas, la comunidad en conjunto, en este caso impulsada mayormente por jóvenes, puede ejercer una pedagogía transformadora y constante, con miras a mejorar la calidad de vida de todos y a generar apego de los jóvenes a su comunidad. Pac Gol ha aunado discursos y perspectivas criticas comunes que dan cuenta del arraigo de sus miembros con la organización, que se observa en la práctica y se ve reforzado por la historia común.

\section{Consideraciones finales}

La MFC se constituye como un medio capaz de proporcionar herramientas a sus jóvenes, en pos de sus desarrollos personales, por un lado, y en cuanto al fortalecimiento de capital social comunitario desde lo colectivo, por otro. Sus trayectorias identitarias se fortalecen individual y colectivamente en un proceso interrelacionado, en el que el desarrollo de cada una alimenta el crecimiento de la otra.

La dimensión individual presenta sujetos que se conforman con pautas críticas ante su propio entorno, con capacidad autónoma y de liderazgo.

La dimensión colectiva, en tanto, presenta un grupo-equipo, que no se limita a la asimilación de valores asociativos meramente anclados a la actividad deportiva vista como ocio, y que puede ser atribuible incluso a la práctica de un fútbol lejano de modelos populares de educación, sino que se extiende a diversas esferas de la vida del joven, y se hace latente desde un discurso común hacia el exterior, distinguiéndose como colectivo. El capital social comunitario adquirido es producto del anclaje educativo que se despliega a raíz de esta actividad deportiva que representa una pasión común para todos. Se presencia desde esta actividad una reapropiación tanto de los cuerpos, de las conciencias participativas como de los espacios sociales comunes al barrio.

Este elemento no ha sido suficientemente abordado por la academia ni tampoco por las políticas sociales. La necesidad de que 
El fútbol callejero como herramienta educativa de transformación social en la comuna de Pedro Aguirre Cerda: organización Pac Gol / Gutiérrez

la autogestión, que caracteriza y da vida a organizaciones como PAC Gol, sea apoyada por los estamentos recién mencionados, sin entrar en lógicas asistencialistas sino que desarrollistas, permitiría cambios sociales que probablemente superarían el nivel local y microsocial.

El anclaje a los movimientos sociales, que han puesto en "entredicho" las identidades individuales y colectivas, puede visualizar un interesante medio o canal de difusión desde las bases de la sociedad civil. Extrapolar su desarrollo y empoderamiento local en el plano sociocultural hacia un nivel macro de la estructura social se torna urgente en estos tiempos en que el individualismo predomina con fuerza en ámbitos como la educación o el laboral, entre otros. Una educación transformadora, si bien se hace explícitamente necesaria en barrios populares y vulnerables, también lo es en todo los estratos del país, para combatir en algún grado la segregación sociocultural que reposa en la estructura social sobre la base del crecimiento económico sostenido pero profundamente inequitativo.

La clave de transformaciones sociales efectivas está en una educación que sepa realizar una adecuada lectura de las juventudes y sus motivaciones. El potencial de ellos se exprime en la medida en que se ancle a las pasiones artísticas, deportivas y culturales con sello colectivo, y no encasillándolos bajo imaginarios de éxito preestablecidos por lógicas de mercado guiadas por el peso de estereotipos. Este fútbol alternativo ofrece desde "la misma calle" una oportunidad para volver a reconocernos y crecer desde lo colectivo.

El sujeto de estudio en la presente investigación se centró en adolescentes; sin embargo, cabe destacar que la mayoría de los jóvenes llevaba años en el circuito y que la gran mayoría del resto de los participantes son niños. Esto da cuenta de la importancia de un trabajo que cuide la infancia y contenga en sí espacios de apoyo afectivo, considerando la vulnerabilidad que se aloja en los diversos espacios cotidianos, tanto como el colegio, el hogar o el barrio. 
Por último, cabe destacar cómo los procesos profundos de cambio social pueden verse mayormente facilitados cuando son impulsados por sujetos que viven y habitan los propios territorios, en comparación a cuando vienen desde instituciones privadas o estatales desde "afuera", y no consagran su real impacto desde lógicas academicistas y teóricas, sino que en la praxis, abordada desde el cuerpo como primer territorio y las emociones; resignificándolas en un terreno político donde pueden ser disputadas. El siglo XXI exige a la especie humana, de forma imperiosa, un cambio de conciencia que implique el cese de las guerras, el extractivismo, el cambio climático y el patriarcado; y la incorporación real, integral e interseccional de todas estas luchas ve un medio idóneo en la reapropiación de las disciplinas artísticas (deportivas, musicales, teatrales), vistas por y para el desarrollo de las mismas personas y las comunidades, y no bajo una mirada de mero consumo cultural.

\section{Bibliografía}

Aguirre, A y Pinto, M (2006). Asociatividad, Capital social y Redes sociales. Revista Mad, 15, Departamento de Antropología Universidad de Chile.

Bourdieu, P. (2000). Las formas del capital: capital económico, capital cultural y capital social, en P. Bourdieu, Poder, derecho y clases sociales. Bilbao: Desclee de Brouwer.

Butler J. (1990). El género en disputa. el feminismo y la subversión de la identidad. Barcelona: Paidos Ibérica.

Cabrera, V. (2013). Planes sociales y participación comunitaria: Estrategias desde la educación social, en Para un Chile más justo: Ideas para fortalecer las regiones y comunas.

Campos \& De Ferrari. (2017). Se juega como se vive ¿Qué selección de fútbol para que Chile queremos? Revista Mensaje. El futuro de Chile. Prioridades políticas.

Carrión, F. (2016). El fútbol coloniza el espacio social, en R. Soto Lagos y O. Fernández Vergara (Comps.), en ¿Quién raya la cancha? Visiones, tensiones y nuevas perspectivas en los estudios socioculturales del deporte en Latinoamérica. Buenos Aires: Clacso.

CASEN. (2015). Ampliando la mirada sobre la pobreza y la desigualdad. Encuesta de Caracterización Socioeconómica Nacional. 
El fútbol callejero como herramienta educativa de transformación social en la comuna de Pedro Aguirre Cerda: organización Pac Gol / Gutiérrez

Santiago de Chile: Ministerio de desarrollo social, Subsecretaria de evaluación social.

Chigol. (2014). ¿Qué es Chigol? Recuperado de http://futbolchigol.cl/ web/que-es-chigol/

Coleman, J. (1990), Foundations of Social Theory. Cambridge, Mass.: Belknap Press.

Côté, J. E. \& Levine, C. (2002). Identity formation, agency, and culture. A social psychological synthesis. Hillsdale, NJ: Lawrence Erlbaum.

Côté, J. E. \& Levine, C. (1997). Students motivations, learning environments, and human capital acquisition: Toward an integrated paradigm of student development. Journal of College Student Development, 38(3): 229-243.

Cruz, E. S. (1998). ¿Hacia dónde va nuestro fútbol? Nueva Sociedad, 154.

Duarte, C. (2005). Cuerpo, poder y placer: Disputas en hombres jóvenes de sectores empobrecidos. Castalia. Revista de Psicología Universidad Academia de Humanismo Cristiano, 6(9): 7184.

Duarte C. (2000). ¿Juventud o juventudes? Acerca de cómo mirar y remirar a las juventudes de nuestro continente. Última Década, $8(13)$.

Duarte, C. (1994). La resistencia de los jóvenes en un país capitalista pobre y dependiente. Revista Pasos, 53, 5-21.

Durán, L. J. y Jiménez, P. J. (2006) Fútbol y Racismo: un problema científico y social. International Journal of Sport Science, 2(3), 68-94.

Durston, J. (2000) ¿Qué es el capital social comunitario? Santiago de Chile: Políticas Sociales, CEPAL.

Echeverría, G. (2005) Análisis cualitativo por categorías. Apuntes docentes de metodología de investigación. Santiago de Chile: Universidad Academia de Humanismo Cristiano, Escuela de Psicología.

El Ciudadano TV. (2016). PAC Gol: fútbol callejero fútbol del pueblo. Recuperado el 8 de noviembre 2017 de: https://www.youtube.com/watch?v=seg_zhDmmoU\&t=1052s)

Fernandez, Y. \& Vergara, C. (2016). Mujeres que van de frente: prácticas sociales y aguante en las hinchas del club Club santiago Wanderers de Valparaiso, parte II, en ¿Quién raya la cancha? Visiones, tensiones y nuevas perspectivas en los estudios socioculturales del deporte en Latinoamérica. Buenos Aires: Clacso. 
Freire, P. (1997). Pedagogía de la autonomía. Buenos Aires: Siglo veintiuno editores.

Freire, P. (1968). Pedagogía del oprimido. México: Biblioteca Nueva

Giménez, G. (1997). Materiales para una teoría de las ciencias sociales. Frontera Norte, 9(18).

Goffman, E., Perrén, H. B. T. \& Setaro, F. (1971). La presentación de la persona en la vida cotidiana (Vol. 60). Buenos Aires: Amorrortu.

Gómez, I. (2015). La filosofía de la educación de Paulo Freire. Revista Internacional de Educación para la Justicia Social, 4(1).

González, J. L. y Fernández, D. (2003). Racismo, discriminación y prejuicio, en I. Fernández, S. Ubillos, E. Zubieta y D. Páez (coords.), Psicología social, cultura y educación (pp. 798804). España: Pearson Educación.

Giddens, A. (1995). Modernidad e identidad del yo. El yo y la sociedad en la época contemporánea. Barcelona: Península.

Giddens, A. (2008). Consecuencias de la modernidad. Alianza.

Gutiérrez, P. (2005). Neoliberalismo, subjetividad y formación de psicólogos en Chile. Castalia: Revista de Psicología de la Academia, 9.

Hall, S. y Dugay, P. (2003). Cuestiones de identidad cultural. Buenos Aires-Madrid; Amorrortu.

Hernández, R. (2010). Metodología de la investigación. Quinta edición: Mc Graw Hill.

Joignant, A. (2012). Habitus, campo y capital: Elementos para una teoría general del capital político. Revista Mexicana de Sociología, 74(4), 587-618.

Lemuñir, J. L. (2017). Nuestra Victoria. A 60 años de la toma de terrenos de la población La Victoria. Relatos de un poblador. Cinco Ases.

Martínez, G. I. (2015). La filosofía de la educación de Paulo Freire. Revista internacional de Educación para la justicia social (RIEJS), 4(1), 55-70.

Matus, C., Vilanova, A. \& Puig, N. (2016). Los clubes deportivos chilenos y su presencia en el desarrollo deportivo local, en R. Soto Lagos y O. Fernández Vergara (Comps.), ¿Quién raya la cancha? Visiones, tensiones y nuevas perspectivas en los estudios socioculturales del deporte en Latinoamérica. Buenos Aires: Clacso.

Moreira, V. (2016) Una mujer en campo masculino y la identificación de género en el proceso de producción del conocimiento an- 
El fútbol callejero como herramienta educativa de transformación social en la comuna de Pedro Aguirre Cerda: organización Pac Gol / Gutiérrez

tropológico, parte II, en R. Soto Lagos y O. Fernández Vergara (Comps.), ¿Quién raya la cancha? Visiones, tensiones y nuevas perspectivas en los estudios socioculturales del deporte en Latinoamérica. Buenos Aires: Clacso.

Movimiento de Fútbol Callejero. (s.f.). Metodología. Recuperado de http://movimientodefutbolcallejero.org/fútbol-callejero/ metodologia/

Páez, D. (2003). Psicología social, cultura y educación. Pearson Educación.

Paiva, V. P. (1982). Paulo Freire y el nacionalismo desarrollista. México: Extemporáneos

PAC Gol. (2017). ¿QQuiénes somos? Recuperado el 27 de septiembre de 2017 de http://www.pacgol.cl/quienes-somos/.

Pérez García, S. (2001). Paulo Freire: educación e ideología. México: Toma y Lee Editorial.

Pérez Luna, E, y Sánchez Carreño, J. (2005). La educación comunitaria: Una concepción desde la Pedagogía de la Esperanza de Paulo Freire. Revista Venezolana de Ciencias Sociales, 9(2), 317-329.

Plascencia, J. (2005). Tres visiones sobre capital social: Bourdieu, Coleman y Putnam. Acta republicana: política y sociedad, 4(4).

Preciado, P. B. (2016). El colegio y el ámbito doméstico están idealizados pero son dos de los espacios más violentos. Entrevista publicada en El Diari de lÉducació.

Putnam, R. (1993). Social Capital and PublicLife. American Prospect. $4(13)$.

Scott, J. (1990). El género: una categoría útil para el análisis histórico, en Nash y Amelang (eds.), Historia y género: las mujeres en la Europa moderna y contemporánea. Valencia: Alfons el Magnanim.

Sub Secretaria de Desarrollo Regional y Administrativo. (2014). Fútbol callejero como herramienta de transformación social. Recuperado el 25 de septiembre de 2017 de https://www. youtube.com $/$ watch? $\mathrm{v}=\mathrm{Vd} 0 \mathrm{xpiparew} \& \mathrm{t}=2 \mathrm{~s}$ )

Stuart, H. \& Du Gray, P. (2003). Cuestiones de identidad cultural. Buenos Aires: Amorrortu.

Urzúa, G., Álvarez, F. y Gaete, T. (2014). El fútbol, en tanto práctica psicosocial, con posibilidad de intervención, en post de la (re) construcción de desarrollo comunitario. Liminales. Ecritos sobre psicología y sociedad/ Universidad Central de chile, 1(5). 
Valenzuela, C. (2012). Capital social en organizaciones juveniles chilenas: la importancia de la confianza y la solidaridad en pares. En R. Israel y M. Villegas (Eds.). Reflexiones sobre la sociedad chilena, (pp.175-196). Santiago de Chile: Ril editories.

Vásquez, C. (2012). Desarrollo y bienestar: Los grandes desafíos de Chile del Bicentenario, en R. Israel \& M. Villagran, Reflexiones sobre la sociedad chilena. Una mirada desde la universidad (pp. 121-137). Santiago de Chile: RIL Editores.

Vélez, B. (2016). Una socio-antropóloga interroga el fútbol y el género, parte II, en R. Soto Lagos y O. Fernández Vergara (Comps.), ¿Quién raya la cancha? Visiones, tensiones y nuevas perspectivas en los estudios socioculturales del deporte en Latinoamérica. Buenos Aires: Clacso. 